\title{
A Wavelet Neural Network Forecasting Model Based On ARIMA
}

\author{
Wang Bin*, Hao Wen-ning, Chen Gang, He Deng-chao, Feng Bo \\ PLA University of Science \&Technology \\ Nanjing 210007, China \\ e-mail: lgdwangbin@163.com
}

\begin{abstract}
Stock index series is Non-stationary, Nonlinear and factors with impact on stock index fluctuation are complex, a time series forecasting model combined ARIMA model and wavelet neural network is presented. The combined model uses BP neural network as the main framework, uses wavelet basis function instead of transfer function in the network, also add some inner factors of the time series mining by ARIMA model, as the part impute of Wavelet Neural Network. So it is more scientific and rational that using inner factors and external other factors. The last simulate experiment shows that the wavelet neural network forecasting model based on ARIMA has higher accuracy than ARIMA model or BP network.
\end{abstract}

Keyword-ARIMA model; Wavelet Neural Network; time series; BP Neural Network

\section{I.QUESTIONS}

Stock index sequence is a special time series. Observing the Shanghai composite index sequence diagram, we know that the time series is non-stationary, and the volatility of the sequence show the non-linear characteristics, also, there are many "singular point" in the stock index fluctuations. In addition to the stock index time series consisting of the internal law of factors that affect stock volatility, there include many social factors such as the national macro-economic policies, financial market crisis, international political issues and market rumors. [1]

Viewing from the literature home and abroad, there are many model types forecasting the volatility of the stock index. According to the different modeling theories, these predictive models can be divied into two broad categories: one is the traditional volatility forecasting model based on statistical principles, the more popular and representative model include the ARIMA model and the SV model; the other prediction model is based on neural network, gray theory and support vector machine. The prediction model based on statistical principles only consider internal rule changes in the time sequence, which cannot indicate the time sequence influenced by multiple indicators and the model with a fixed relationship is difficult to accurately forecast the stock index.[2,3] The neural network forecasting model have multiple indicators and independent learning, self-adjustment function, but the model does not support the large sample, multi-level classification problems.

\section{II.STUDIES}

Many scholars study detailed and in-depth the forecast of the stock index sequence. The reference [4] combine the gray forecasting model and Markov model to form the combined forecasting model, and the experiment proved that the combination model has a higher accuracy than single forecasting model. The reference [5] made stock price prediction using BP neural network with adjustable weighting factors and profits, and got a better prediction. The reference [6] forecast the Shanghai Composite Index using the added bee colony algorithm fuzzy neural network. The reference [7] introduced incremental learning and abandonment data method in SVM, and applied it to the stock price prediction. The reference [8] combined ARIMA model and SVM forecasting model, and proposed a combination model for stock price forecasting, which conclusions is superior to the single ARIMA model or SVM model. Many scholars now pay more attention to how to effectively combine the existing single predictor model, and establish a combination prediction model to improve the accuracy of the forecast and obtain a more favorable forecast.

Neural network can approximate any rational function to arbitrary precision, and therefore has the ability to express arbitrary nonlinear relations and to learn, the method is applicable to a wide range; ARIMA model is a commonly used class of stochastic time series model, using the extrapolation mechanism to describe the changes in the time series, and specializes in the influencing rule in time series; wavelet transform has become a powerful tool of Nonlinear Science, and has multi-resolution characteristics and the ability to characterize the local signal characteristics in the time-frequency domain, making wavelet transform becoming more and more applicated in the field of signal analysis and forecast. Therefore, the wavelet neural network is constituted here combining the good frequency localization characteristics of neural wavelet and earning characteristics of neural network together; at the same time taking advantage of the internal changes of ARIMA model analysis sequence, combining the wavelet neural network to establish a combined prediction model for stock Prediction of the index. $[9,10]$

\section{III.WAVELET NEURAL NETWORK PREDICTION MODEL CONSTRUCTION BASED ON ARIMA}

The wavelet neural network prediction model takes BP neural network as the main framework and analysis 
sequence inherent associated indicators of ARIMA model as part of the BP network input, while introduce other factors that affect stock index sequence fluctuations as the input of BP network, combining with wavelet basis function as BP network transfer function. The combination forecasting model fully consider the internal and external factors that affect the sequence fluctuations, taking wavelet basis function as the transfer function of the network to overcome the network falling into a local sub-optimal solution in the training process and enable the network to achieve the best convergence effect.

\section{A. Wavelet neural network design}

Wavelet analysis transform can analysis local features of signals by the transform wavelet basis function, and has the ability to choose the direction of signal in the two-dimensional case. the basic idea of combining the advantages of neural network and wavelet analysis is based on BP neural network topology, takes as wavelet basis function as the transfer function of the hidden layer nodes, corresponding weights and threshold values replaced by the scale factor and shift factor of wavelet function. It is a neural network with a signal in the traveling forward while propagation while error traveling back.

Wavelet neural network model adopt a three-tier structure of wavelet neural network, namely input layer, hidden layer and output layer. The model structure is shown in Figure 1:

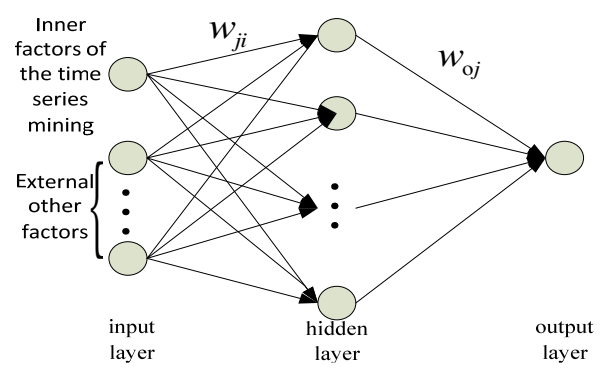

Figure 1 The Wavelet Neural network model

When input time series is $X_{t}(\mathrm{t}=1,2 \ldots, \mathrm{k})$, hidden layer output is calculated as follows:

$$
\mathrm{h}(\mathrm{j})=h_{j}\left(\frac{\sum_{i=1}^{k} \omega_{i j} x_{i}-b_{j}}{a_{j}}\right), j=1,2, \ldots l
$$

$h(j)$ is the value of the output of the jth node of hidden layer nodes; $\omega_{i j}$ connection weights for the input layer and the hidden layer; $b_{j}$ translates as wavelet basis function factor; dilation factor as a function of the wavelet basis; $h_{j}$ wavelet basis function. The wavelet neural network output layer formula:

$$
\mathrm{y}(\mathrm{k})=\sum_{i=1}^{l} \omega_{o j} h(i), k=1,2, \ldots m
$$

Among them: the hidden layer to the output layer weights; as the output of the first hidden layer nodes; hidden layer nodes for the output layer nodes. The wavelet neural network with gradient correction method to fix the network weights and parameters of wavelet basis functions, thereby enabling the output of the wavelet neural network prediction closer and closer to the desired output. Wavelet neural network algorithm training steps:

Step1: Select the appropriate wavelet;

Step2: initiates dilation factor $a_{k}$, of the random wavelet function, translation factors and the network connection weights heavy with, and set up the network learning rate $\eta$;

Step3: Enter the training samples to the network, and then calculate the network output and calculate the network output and the desired output deviation;

Step4: correct network weights and wavelet function parameters through e, approaching the network prediction values to the expectations;

Step5: determine whether converge or achieve the maximum iteration number of network settings, if converge achieve the maximum number, then stop, otherwise return to Step 3;

\section{B. ARIMA time series prediction model}

The general form of ARIMA (Auto regressive integrated moving average) model: $\operatorname{ARIMA~}(p, d, q)$

Where $p$ is the order of the auto regressive model; $D$ is order by phase difference; $\mathrm{q}$ is the order of the moving average. When the time sequence after d-order differential becomes smooth sequence, create the $\operatorname{ARMA}(p, q)$ model. The model parameters are as follows:

$$
\begin{aligned}
& X_{\mathrm{t}}=\varphi_{1} X_{\mathrm{t}-1}+\varphi_{2} X_{\mathrm{t}-2}+\ldots+\varphi_{\mathrm{p}} X_{\mathrm{t}-\mathrm{p}}+\mathrm{u}_{\mathrm{t}}-\theta_{1} \mathrm{u}_{\mathrm{t}-1}-\theta_{2} \mathrm{u}_{\mathrm{t}-2} \\
& -\ldots-\theta_{\mathrm{q}} \mathrm{u}_{\mathrm{t}-\mathrm{q}}
\end{aligned}
$$

Time series $X_{t}$ is a linear combination of its current and prior deviation term $\left(u_{t}, u_{t-1}, \ldots, u_{t-q}\right)$ and the pre-value $\left(X_{t-1}, X_{t-2}, \ldots, X_{t-p}\right)$. Regression coefficients $\left(\varphi_{1}, \varphi_{2}, \ldots \varphi_{p}\right)$; moving average coefficient $\left(\theta_{1}, \theta_{2}, \ldots, \theta_{q}\right)$, they are the model parameters to be estimated. Establish the ARIMA model process as shown in Figure 2:

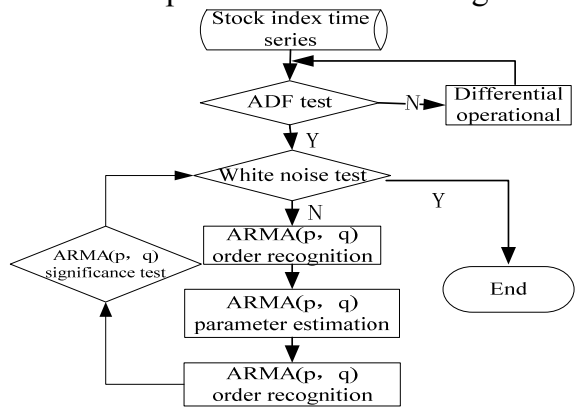

Figure 2 ARIMA (p, d, q) model building process

Test the stationarity of indice time series. If it is a non-stationary time series, do the difference computing and get a smooth sequence, and then do the white noise test, if the sequence is a white noise sequence, then it is a random sequence, and do not need to do model Analysis. The smooth non-white noise sequence firstly identify the model, to identify the type and order of the model (i.e., to determine the values of the parameters p, q) using successive heuristics. Then use a nonlinear least squares method to estimate parameters (i.e., to determine the 
values of $\left.\theta_{i}, \varphi_{i}\right)$; finally, test fitting model based on samples, in order to find certain inadequacies. If parameters of estimated model cannot pass significant test, not all roots of auto regressive characteristic equation are outside the unit element, or residual sequence cannot be approximated as a white noise sequence, you should return to the first step and identify the model. If the above two problems did not exist, you can accept the established model.

\section{ARIMA-based design of wavelet neural network prediction model}

Prediction model based on ARIMA wavelet neural network, firstly create a time series ARIMA model, get the linear relationship of the current value $X_{t}$, the past value $\left(X_{t-1}, X_{t-2}, \ldots, X_{t-p}\right)$ and the error value $\left(u_{t}, u_{t-1}, \ldots, u_{t-q}\right)$, then get the law of the time series variation. At the same time, use of economic theory and various statistical methods to gain index sequence fluctuation change affects large technical indicators (set to $T_{1}, T_{2}, \ldots, T_{m}$ ); finally obtain input variables of wavelet neural network. Its structure can be represented as:

Inner factors of the time series mining

$q=f(\overbrace{u_{t}, u_{t-1}, \ldots, u_{t-q}, X_{t-1}, X_{t-2}, \ldots, X_{t-p}}$,

External other factors

$\overbrace{\mathrm{T}_{1}, \mathrm{~T}_{2}, \ldots, \mathrm{T}_{\mathrm{m}}}$ )

$\mathrm{f}$ is wavelet neural network, $\mathrm{q}$ is the predicted value. The Wavelet Neural networks adopt the 3.1 three tie network architecture. The number of neurons in the input layer network

$u_{t}, u_{t-1}, \ldots, u_{t-q}, X_{t-1}, X_{t-2}, \ldots, X_{t-p}, T_{1}, T_{2}, \ldots, T_{m}$; numbe $\mathrm{r}$ of neurons in the hidden layer is determined by the formula $(m+n)^{1 / 2}+\alpha, \mathrm{m}$ input layer neuron number, $\mathrm{n}$ is the number of neurons in the output layer, $\alpha$ a number between 1-10 ; the output layer neural unit numbers for the predicted time sequence, and is set to 1 here.

\section{IV.SIMULATION}

\section{A. Description of Experiment}

The experimental environment: Intel CPUs 2 G RAM, 250G hard drive, Windows XP operating system. Mat lab software and SPSS statistical analysis software to verify validity of combination forecasting model. Experimental data was selected from March 2008 to June 2012, the Shanghai stock composite index daily historical data, and collect indicators that have a greater impact on stock volatility, they are BISA6 (stock index the good offline) on the 6th, PSY12 (stock index psychological line in the 12th), MA5 (stock index 5 days moving average indicator), W \& R1 (stock index on the 1st William

\begin{tabular}{llllllll}
\hline Data & $\begin{array}{l}\text { Truth } \\
\text { value }\end{array}$ & $\begin{array}{l}\text { CM } \\
\text { P-value }\end{array}$ & $\begin{array}{l}\text { CM } \\
\text { R-error }\end{array}$ & $\begin{array}{l}\text { ARIMA } \\
\text { P-value }\end{array}$ & $\begin{array}{l}\text { ARIMA } \\
\text { R-error }\end{array}$ & $\begin{array}{l}\text { BPNN } \\
\text { P-value }\end{array}$ & $\begin{array}{l}\text { BPNN } \\
\text { R-error }\end{array}$ \\
\hline $2012-6-1$ & 2373.4 & 2380.1 & 0.28 & 2369.02 & 0.2 & 2399.7 & 1.10 \\
$2012-6-4$ & 2308.6 & 2326.9 & 0.77 & 2370.44 & 2.67 & 2339.5 & 1.34 \\
$2012-6-5$ & 2311.9 & 2315.9 & 0.11 & 2370.42 & 2.53 & 2269.9 & 1.81
\end{tabular}
$1 /(1+e-x)$.

\section{B. Experimental procedure and results}

1) Establishment of ARIMA model. is: $X_{t}=-2.382 X_{t-1}-2.803 X_{t-2}$.

2) Training of wavelet neural network. one-dimensional wavelet function as:

$$
\mathrm{f}=\exp \left(-t^{2} / 2 * \cos (1.75 * t)\right)
$$

$3)$ Predictions of combined model Specific forecast results are shown in Table 1: that impact fluctuations in the stock indexes, such as the CPI, social sudden situation, monetary policy, quantify the text class indicators to 11 external impact indicators. Firstly analyze stock index series, establish ARIAM model to estimate the parameters $\varphi_{1}, \varphi_{2}, \ldots \varphi_{p} \theta_{1}, \theta_{2}, \ldots, \theta_{q}$ of the model. Calculate parameters and the current value of the error term with the established model, and combine larger targets which have larger impact on stock index volatility to form the input of the wavelet neural network. Continuously computing the historical data by imputing to the wavelet network, and identify the network weights which make network converge. Finally, use the stable network to predict the next sequence of the 11-stock index, get the stock index predictive value, and make analysis with prediction results. In order to verify the descriptive of the experimental results, use ARIMA model to predict stock index sequence volatility and BP network which is the same as wavelet neural network structure to do the prediction, among which the transfer function of the neurons of BP network adopt Sigmoid function: $\delta(\mathrm{x})=$

Do the first difference of the time series sequence $\nabla X_{t}=X_{t}-X_{t-1}$ then find the differential sequence is smooth through self-correlation and partial correlation test, that is, determine the parameter $\mathrm{d}=1$; After gradual search the established parameter ARIMA model is ARIMA $(2,1$, $0)$; The parameter form of the model

Adopt a three-tier network topology, the input layer with 13 neurons, among which 11 are external impact mapping, internal law change of the sequence found 2 indexes ; in hidden layer the neurons are 9, in output layer neural elements number is 1 . The maximum number of iterations of the network is 1500 , learning rate of wavelet neural network is set to 0.1 , minimum of the expect error is set to 0.01 . The chosen wavelet is Morlet

After obtaining trained prediction model, make the Shanghai Composite Index from June 1, 2012 to 15 as the test set, get the predicted results, and do simulation experiments taking advantage of the ARIMA model and BP neural network, get the corresponding predicted values. 


\begin{tabular}{llllllll}
$2012-6-6$ & 2309.6 & 2288.8 & 0.90 & 2370.3 & 2.62 & 2357.1 & 2.06 \\
$2012-6-7$ & 2293.1 & 2269.9 & 1.04 & 2370.31 & 3.37 & 2257.3 & 1.56 \\
$2012-6-8$ & 2281.5 & 2251.5 & 1.34 & 2370.32 & 3.89 & 2229.5 & 2.27 \\
$2012-6-11$ & 2305.9 & 2299.5 & 0.27 & 2370.32 & 2.79 & 2275.7 & 1.31 \\
$2012-6-12$ & 2289.8 & 2287.6 & 0.10 & 2370.32 & 3.51 & 2329.6 & 1.74 \\
$2012-6-13$ & 2318.9 & 2289.3 & 1.24 & 2370.32 & 2.21 & 2271.1 & 2.06 \\
$2012-6-14$ & 2295.9 & 2284.5 & 0.46 & 2370.32 & 3.24 & 2261.3 & 1.51 \\
$2012-6-15$ & 2306.8 & 2310.7 & 0.17 & 2370.32 & 2.75 & 2349.1 & 1.83 \\
\hline
\end{tabular}

\section{Experimental Analysis}

Observing Figure 3 and Table 1, ARIMA prediction model can only be for a short-term prediction. With the prediction days increasing, the prediction result remains unchanged, and the prediction error is large and average error of 11 prediction values is $2.707 \%$, for the reason that ARIMA model only do extrapolated prediction from the sequence itself and it cannot be sensitive to perceived external impact indicators for sequence, so it is difficult to accurately predict the turning point. Accuracy of BP prediction network has a larger ascension than ARIMA, the average relative error of 11 times forecast results is $1.69 \%$ in Table 1, but Figure 3 shows that the BP network predictive value fluctuates over real value curve, showing a single BP network prediction model is not very stable; BP neural network has a strong perception of the impact indicators, and it can establish the relationship between the timing and several indicators. However, due to the convergence of the BP neural network, and easiness to fall into local optimum, it can affect the predicted effect.

Wavelet neural network prediction model based on the ARIMA has a big increase in prediction accuracy than Single ARIMA model and BP neural network forecasting model, the average value of 11 times forecast relative error is $0.607 \%$, with a smaller error fluctuation range of the predicted values. And maximum relative error is about $1.3 \%$, which compared with the BP network model has some improvement in terms of stability. The combination forecasting model combines the advantages of the two single model, fully consider the internal and external influencing factors that impact stock index fluctuations, making the accuracy of combination forecasting model greatly improved, and with the wavelet basis function being the transfer function of the BP neural network to overcome the network into local time advantages, it makes better convergence of the network in the training process, and enhance the model fitting degree of timing, at the same time speed up the convergence.

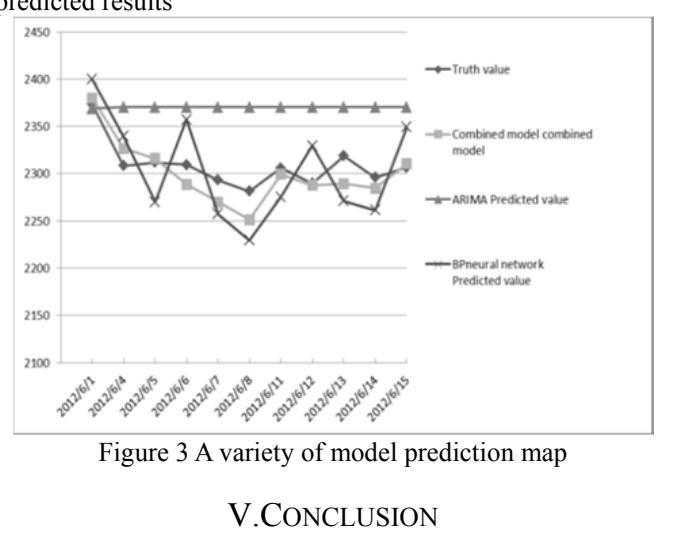

In this paper, due to the numerous and complex factors of fluctuations of the stock index time series, we establish a wavelet neural network prediction model based on ARIMA, with internal law of sequence mining by the model as part of the input, and the wavelet basis function as a transfer function in the network. Simulation results show that the combination model has a high accuracy compared with the ARIMA model or the BP network model thus has a good value of use. But the combination model has complex calculation steps, and the determination of the wavelet basis function networks and network nodes need to be determined based on experience, so this method has some limitations. In addition, non-textual factors to determine the impact factor of the stock index sequence are the next step of the research.

\section{REFERENCES}

[1] Cai Feng, Zeng Feng-zhang. "ANN-ARMA Model for Forecasting Product Consumption Based on Non-Stationary Time Series",Transactions of Beijing institute of technology, 2007, 27(3);

[2] Xie Bing, Dai Sheng, Xie Ke-fan. "Forecast of Industrial Stock Index Based on Neural Network and Genetic Algorithm", Hubei University.2004, 11;

[3] Wu Ming-feng, Jiang Xun. "Forecasting Model Based on Fuzzy Time Series", Value engineering, 2008。

[4] Chen Hai-ming,Li Dong, "The application of grey forecast model in stock price",Sciences research management,203,(3):70-74;

[5] Watanbe H, Chakraborty, B.Chakrabortyj, "Approach for Stock Price Trend Forecasting from Multivariate Time Series", Second International Conference on Information and Control, 2007 (9);

[6] Huang, Fu-Yuan. "Integration of an Improved Particle Swarm Algorithm and Fuzzy Neural Network for Shanghai stock Market Prediction", International Conference on Power Electronics and Intelligent Transportation System,2008(8):242-247;

[7] H.Nakayama, A.Hattori. "Incremental learning and forgetting in 
RBF networks and SVM Witn applications to financial problems", Knowledge-based intelligent information and engineering systems, 2003, 2773(1):1109-1115;

[8] Pai,C.Lin. "A hybrid ARIMA and support vector machines model in stock price forecasting", Omega, 2005, 33(6):497-505;

[9] Shen Wei. "Research on Method and Application of Building Forecasting Models for Stock Index Fluctuation “,2011, 6;

[10] Li Zhi. "Wavelet theory and economic and financial sequential application research",2007 